\title{
Quantum Mechanics is About Quantum Information
}

\author{
Jeffrey Bub \\ Department of Philosophy, University of Maryland, \\ College Park, MD 20742 \\ (E-mail: jbub@carnap.umd.edu)
}

November 1, 2018

\begin{abstract}
I argue that quantum mechanics is fundamentally a theory about the representation and manipulation of information, not a theory about the mechanics of nonclassical waves or particles. The notion of quantum information is to be understood as a new physical primitive_-just as, following Einstein's special theory of relativity, a field is no longer regarded as the physical manifestation of vibrations in a mechanical medium, but recognized as a new physical primitive in its own right.
\end{abstract}

\section{Introduction}

In several places [9, 10, 11], Cushing speculates about the possibility of an alternative history, in which Bohm's theory [4] 16] is developed as the standard version of quantum mechanics, and suggests that in that case the Copenhagen interpretation, if it had been proposed as an alternative to a fully developed Bohmian theory, would have been summarily rejected. I quote from [10 pp. 352-353]:

... we can fashion a highly reconstructed but entirely plausible bit of partially 'counterfactual' history as follows (all around 1925-1927). Heisenberg's matrix mechanics and Schrödinger's wave mechanics are formulated and shown to be mathematically equivalent. Study of a classical particle subject to Brownian motion ... leads to a classical understanding of the already discovered Schrödinger equation. A stochastic mechanics underpins this interpretation with a visualizable model of microphenomena and, so, a realistic ontology remains viable. Since stochastic mechanics is quite difficult to handle mathematically, study naturally turns to the mathematically equivalent linear Schrödinger equation. Hence, the Dirac transformation theory and an operator formalism are available as a convenience for further development of the mathematics to provide algorithms for calculation. $\cdots$

A Bell-type theorem is proven and taken as convincing evidence that nonlocality is present in quantum phenomena. A no-signalling theorem for 
quantum mechanical correlations is established and this puts to rest Einstein's objections to the nonseparability of quantum mechanics. ... This could reasonably have been enough to overcome his objections to the nonlocal nature of a de Broglie-Bohm interpretation of the formalism of quantum mechanics. Because the stochastic theory is both nonlocal and indeterministic, whereas the de Broglie-Bohm model is nonlocal only and still susceptible to a realist interpretation, Einstein might have made the transition to the latter type of theory.

That is, these developments, that could, conceptually and logically, have taken place around 1927, could have overcome the resistance of Einstein and of Schrödinger to supporting a de Broglie-Bohm program. ... Bohm's interpretation would certainly have been possible in 1927. These models and theories could be generalized to include relativity and spin. The program is off and running. Finally, this causal interpretation can be extended to quantum fields.

So, if, say, in 1927, the fate of the causal interpretation had taken a very different turn and been accepted over the Copenhagen one, it would have had the resources to cope with the generalizations essential for a broadbased empirical adequacy. We could today have arrived at a very different world view of microphenomena. If someone were then to present the merely empirically equally as adequate Copenhagen version, with all of its own additional counterintuitive and mind-boggling aspects, who would listen? ... However, Copenhagen got to the top of the hill first and, to most practicing scientists, there seems to be no point in dislodging it.

Cushing's broader and very interesting thesis was that the successful theories that philosophers of science analyze as case-studies are themselves contingent on historical factors - in particular, the success of the Copenhagen interpretation of quantum mechanics is a matter of historical contingency. I want to play Cushing's counterfactual game for the case of special relativity and compare this with the quantum mechanical case to argue for a very different thesis: the interpretation of quantum mechanics as a theory about the representation and manipulation of information in our world, not a theory about the mechanics of nonclassical waves or particles.

The following discussion is divided into three sections. In 'Principle vs Constructive Theories,' I discuss Einstein's distinction between these two classes of theories, and the significance of his characterization of special relativity as a principle theory. I conclude the section by arguing that, just as the rejection of Lorentz's theory in favour of special relativity (formulated in terms of Einstein's two principles) involved taking the notion of a field as a new physical primitive, so the rejection of Bohm's theory in favour of quantum mechanics - characterized via the Clifton-Bub-Halvorson ( $\mathrm{CBH})$ theorem [7] in terms of three information-theoretic principles-involves taking the notion of quantum information as a new physical primitive. (By 'information' here, I mean information in the physical sense, measured classically by the Shannon entropy and, in quantum mechanics, by the von Neumann entropy.) In 'The CBH Characterization Theorem,' I outline the content of the $\mathrm{CBH}$ theorem. Finally, in 'Quantum Information,' I argue that, just as Einstein's analysis (based on the assumption that we live in a world in which natural processes are subject to certain constraints specified by the principles of special relativity) shows that the mechanical structures in Lorentz's constructive theory (the ether, and the behaviour of electrons in the ether) are irrelevant to a physical explanation of electromagnetic phenomena, so the $\mathrm{CBH}$ analysis (based on the assumption that we live in a world in which there are certain constraints on 
the acquisition, representation, and communication of information) shows that the mechanical structures in Bohm's constructive theory (the guiding field, the behaviour of particles in the guiding field) are irrelevant to a physical explanation of quantum phenomena. You can, if you like, tell a story along Bohmian, or similar, lines (as in other 'no collapse' interpretations) but, given the information-theoretic constraints, such a story can, in principle, have no excess empirical content over quantum mechanics (just as Lorentz's theory, insofar as it is constrained by the requirement to reproduce the empirical content of the principles of special relativity, can, in principle, have no excess empirical content over Einstein's theory).

\section{Principle vs Constructive Theories}

Einstein introduced the distinction between principle and constructive theories in an article on the significance of the special and general theories of relativity that he wrote for the London Times, which appeared in the issue of November 28, 1919 [13]:

We can distinguish various kinds of theories in physics. Most of them are constructive. They attempt to build up a picture of the more complex phenomena out of the material of a relatively simple formal scheme from which they start out. Thus the kinetic theory of gases seeks to reduce mechanical, thermal, and diffusional processes to movements of moleculesi.e., to build them up out of the hypothesis of molecular motion. When we say that we have succeeded in understanding a group of natural processes, we invariably mean that a constructive theory has been found which covers the processes in question.

Along with this most important class of theories there exists a second, which I will call 'principle theories.' These employ the analytic, not the synthetic, method. The elements which form their basis and starting-point are not hypothetically constructed but empirically discovered ones, general characteristics of natural processes, principles that give rise to mathematically formulated criteria which the separate processes or the theoretical representations of them have to satisfy. Thus the science of thermodynamics seeks by analytical means to deduce necessary conditions, which separate events have to satisfy, from the universally experienced fact that perpetual motion is impossible.

Einstein's point was that relativity theory is to be understood as a principle theory. He returns to this theme in his 'Autobiographical Notes' 14 pp. 51-52], where he remarks that he first tried to find a constructive theory that would account for the known properties of matter and radiation, but eventually became convinced that the solution to the problem was to be found in a principle theory that reconciled the constancy of the velocity of light in vacuo for all inertial frames of reference, and the equivalence of inertial frames for all physical laws (mechanical as well as electromagnetic):

Reflections of this type made it clear to me as long ago as shortly after 1900, i.e., shortly after Planck's trailblazing work, that neither mechanics nor electrodynamics could (except in limiting cases) claim exact validity. By and by I despaired of the possibility of discovering the true laws by means of constructive efforts based on known facts. The longer and the more despairingly I tried, the more I came to the conviction that only the discovery of a universal formal principle could lead us to assured results. 
The example I saw before me was thermodynamics. The general principle was there given in the theorem: the laws of nature are such that it is impossible to construct a perpetuum mobile (of the first and second kind). How, then, could such a universal principle be found?

A little later [14 p. 57], he adds:

The universal principle of the special theory of relativity is contained in the postulate: The laws of physics are invariant with respect to the Lorentztransformations (for the transition from one inertial system to any other arbitrarily chosen system of inertia). This is a restricting principle for natural laws, comparable to the restricting principle for the non-existence of the perpetuum mobile which underlies thermodyamics.

According to Einstein, two very different sorts of theories should be distinguished in physics. One sort involves the reduction of a domain of relatively complex phenomena to the properties of simpler elements, as in the kinetic theory, which reduces the mechanical and thermal behavior of gases to the motion of molecules, the elementary building blocks of the constructive theory. The other sort of theory is formulated in terms of 'no go' principles that impose constraints on physical processes or events, as in thermodynamics ('no perpetual motion machines'). For an illuminating account of the role played by this distinction in Einstein's work, see the discussion by Martin Klein in [23].

The special theory of relativity is a principle theory, formulated in terms of two principles: the equivalence of inertial frames for all physical laws (the laws of electromagnetic phenomena as well as the laws of mechanics), and the constancy of the velocity of light in vacuo for all inertial frames. These principles are irreconcilable in the geometry of Newtonian space-time, where inertial frames are related by Galilean transformations. The required revision yields Minkowski geometry, where inertial frames are related by Lorentz transformations. Einstein characterizes the special principle of relativity, that the laws of physics are invariant with respect to Lorentz transformations from one inertial system to another, as 'a restricting principle for natural laws, comparable to the restricting principle for the non-existence of the perpetuum mobile which underlies thermodynamics.' (In the case of the general theory of relativity, the group of allowable transformations includes all differentiable transformations of the space-time manifold onto itself.) By contrast, the Lorentz theory [27], which derives the Lorentz transformation from the electromagnetic properties of the ether, and assumptions about the transmission of molecular forces through the ether, is a constructive theory.

Consider the transition:

Lorentz's constructive mechanical theory of the electrodynamics of moving bodies

$\longrightarrow$ Einstein's principle theory of special relativity

$\longrightarrow$ Minkowski's formulation of Einstein's theory in terms of a non-Euclidean spacetime geometry

Einstein showed that you could obtain a unified treatment of mechanical and electromagnetic phenomena - particles, electrons, light—by extending the idea of Galilean relativity (in a suitably modified form, involving the Lorentz transformation between inertial frames) to both mechanical and electromagnetic phenomena. In Minkowski's formulation of the theory, the relativistic principles are instantiated in a specific nonNewtonian geometry of space-time. In this new framework, rigid bodies are excluded by the symmetry group (i.e., they would transmit signals faster than light) and, strictly speaking, particles (insofar as they are small rigid bodies) are excluded. Instead, the field becomes the basic physical entity, as a new physical primitive. In particular, 
since an electromagnetic wave is not reduced to the vibratory motion of a mechanical medium (as a sound wave is reducible to the notion of air molecules), the ether is no longer required as the medium for the physical instantiation of an electromagnetic field.

Now compare the historical transition:

Lorentz's constructive theory

$\longrightarrow$ Einstein's principle theory

$\longrightarrow$ Minkowski space-time

with the transition in a modified version of Cushing's counterfactual history:

Bohm's constructive theory

$\longrightarrow X$

$\longrightarrow$ Hilbert space quantum mechanics

That is (for comparison with the relativistic case), assume that Bohm's theory was actually developed before Hilbert space quantum mechanics as a solution to some of the experimental difficulties of classical mechanics at the turn of the 20 'th century, and that there was an additional development, something like an Einsteinian formulation of quantum mechanics as a principle theory (the ' $X$ ').

Without the ' $\mathrm{X}$ ' step, the Copenhagen argument for the completeness of Hilbert space quantum mechanics (and the associated rejection of Bohm's theory) in the counterfactual world seems implausible, as Cushing suggests. Similarly, to consider another counterfactual history, we might suppose that (after Lorentz's theory) the special theory of relativity was first formulated geometrically by Minkowski rather than Einstein, as an algorithm for relativistic kinematics and the Lorentz transformation, which is incompatible with the kinematics of Newtonian space-time. Without Einstein's analysis of the theory as a principle theory along the lines sketched above, it seems implausible to suppose that Lorentz's theory would have been dislodged by what would surely have seemed to be merely a convenient (but 'counterintuitive and mind-boggling') algorithm.

In the following section, I argue that the missing ' $X$ ' (in a logical sense) is supplied by the $\mathrm{CBH}$ characterization theorem for quantum theory in terms of three informationtheoretic constraints and that, given this theorem, the relation between quantum mechanics and constructive theories like Bohm's theory should be seen as analogous to the relation between special relativity and Lorentz's theory. Just as special relativity involves a theory of the structure of space-time in which a field is a new physical primitive not reducible to the motion of a mechanical medium (ultimately, to the motion of particles), so quantum mechanics involves a theory of the algebraic structure of states and observables in which information is a new physical primitive not reducible to the behaviour of mechanical systems (the motion of particles or fields).

It should go without saying that I am not comparing the CBH theorem with Einstein's achievement in developing the special theory of relativity. To avoid any such suggestion, which would be ludicrous, let me say what would perhaps be a comparable achievement. Suppose, in a modified version of Cushing's counterfactual history, that in 1927 Bohm's theory was the dominant research paradigm in quantum physics. Suppose (in 1927) that CBH showed that one could dispense with the whole idea of a source-less field in configuration space guiding the motion of particles by deriving the current Hilbert space theory from three information-theoretic constraints, and in terms of this (then new) Hilbert space theory also showed in detail how one could treat various quantum systems, formerly treated in terms of Bohm's theory, in a much simpler way, and in particular brought out the implications of entanglement as a new physical resource that could be exploited to develop novel forms of computation and cryptographic procedures that were impossible classically (cf. $E=m c^{2}$ ). In our actual history, since Hilbert space quantum mechanics and quantum information theory 
are already on the table, the $\mathrm{CBH}$ theorem is hardly more than a footnote to current theory. The purpose in pointing to the analogy is to argue that the relevance of the $\mathrm{CBH}$ theorem to the interpretative debate about Hilbert space quantum mechanics and the significance of constructive mechanical theories like Bohm's theory, is to be understood as similar to the relevance of Einstein's analysis of special relativity as a principle theory to Minkowski's geometric formulation of the theory and Lorentz's constructive mechanical ether theory.

\section{The CBH Characterization Theorem}

The CBH characterization theorem is formulated in the general framework of $C^{*}$ algebras, which allows a mathematically abstract characterization of a physical theory that includes, as special cases, all classical mechanical theories of both wave and particle varieties, and all variations on quantum theory, including quantum field theories (plus any hybrids of these theories, such as theories with superselection rules). So the analysis is not restricted to the standard quantum mechanics of a system represented on a single Hilbert space with a unitary dynamics, but is general enough to cover cases of systems with an infinite number of degrees of freedom that arise in quantum field theory and the thermodynamic limit of quantum statistical mechanics (in which the number of microsystems and the volume they occupy goes to infinity, while the density defined by their ratio remains constant), including the quantum theoretical description of exotic phenomena such as Hawking radiation, black hole evaporation, Hawking information loss, etc. The Stone-von Neumann theorem, which guarantees the existence of a unique representation (up to unitary equivalence) of the canonical commutation relations for systems with a finite number of degrees of freedom, breaks down for such cases, and there will be many unitarily inequivalent representations of the canonical commutation relations. One could, of course, consider weaker mathematical structures, but it seems that the $C^{*}$-algebraic machinery suffices for all physical theories that have been found to be empirically successful to date, including phase space theories and Hilbert space theories [24], and theories based on a manifold [8].

A $C^{*}$-algebra is essentially an abstract generalization of the structure of the algebra of operators on a Hilbert space. Technically, a (unital) $C^{*}$-algebra is a Banach *-algebra over the complex numbers containing the identity, where the involution operation * and the norm are related by $\left\|A^{*} A\right\|=\|A\|^{2}$. So the algebra $\mathfrak{B}(\mathcal{H})$ of all bounded operators on a Hilbert space $\mathcal{H}$ is a $C^{*}$-algebra, with * the adjoint operation and $\|\cdot\|$ the standard operator norm.

In standard quantum theory, a state on $\mathfrak{B}(\mathcal{H})$ is defined by a density operator $D$ on $\mathcal{H}$ in terms of an expectation-valued functional $\rho(A)=\operatorname{Tr}(A D)$ for all observables represented by self-adjoint operators $A$ in $\mathfrak{B}(\mathcal{H})$. This definition of $\rho(A)$ in terms of $D$ yields a positive normalized linear functional. So a state on a $C^{*}$-algebra $\mathfrak{C}$ is defined, quite generally, as any positive normalized linear functional $\rho: \mathfrak{C} \rightarrow \mathbb{C}$ on the algebra. Pure states are defined by the condition that if $\rho=\lambda \rho_{1}+(1-\lambda) \rho_{2}$ with $\lambda \in(0,1)$, then $\rho=\rho_{1}=\rho_{2}$; other states are mixed.

The most general dynamical evolution of a system represented by a $C^{*}$-algebra of observables is given by a completely positive linear map $T$ on the algebra of observables, where $0 \leq T(I) \leq I$. The map or operation $T$ is called selective if $T(I)<I$ and nonselective if $T(I)=I$. A yes-no measurement of some idempotent observable represented by a projection operator $P$ is an example of a selective operation. Here, $T(A)=P A P$ for all $A$ in the $C^{*}$-algebra $\mathfrak{C}$, and $\rho^{T}$, the transformed ('collapsed') state, is the final state obtained after measuring $P$ in the state $\rho$ and ignoring all elements of the ensemble that do not yield the eigenvalue 1 of $P$ (so 
$\rho^{T}(A)=\rho(T(A)) / \rho(T(I))$ when $\rho(T(I)) \neq 0$, and $\rho^{T}=0$ otherwise). The time evolution in the Heisenberg picture induced by a unitary operator $U \in \mathfrak{C}$ is an example of a nonselective operation. Here, $T(A)=U A U^{-1}$. Similarly, the measurement of an observable $O$ with spectral measure $\left\{P_{i}\right\}$, without selecting a particular outcome, is an example of a nonselective operation, with $T(A)=\sum_{i=1}^{n} P_{i} A P_{i}$. Note that any completely positive linear map can be regarded as the restriction to a local system of a unitary map on a larger system.

A representation of a $C^{*}$-algebra $\mathfrak{C}$ is any mapping $\pi: \mathfrak{C} \rightarrow \mathfrak{B}(\mathcal{H})$ that preserves the linear, product, and ${ }^{*}$ structure of $\mathfrak{C}$. The representation is faithful if $\pi$ is one-toone, in which case $\pi(\mathfrak{C})$ is an isomorphic copy of $\mathfrak{C}$. The Gelfand-Naimark theorem says that every abstract $C^{*}$-algebra has a concrete faithful representation as a normclosed *-subalgebra of $\mathfrak{B}(\mathcal{H})$, for some appropriate Hilbert space $\mathcal{H}$. In the case of systems with an infinite number of degrees of freedom (as in quantum field theory), there are inequivalent representations of the $C^{*}$-algebra of observables defined by the commutation relations.

The relation between classical theories and $C^{*}$-algebras is this: every commutative $C^{*}$-algebra $\mathfrak{C}$ is isomorphic to the set $C(X)$ of all continuous complex-valued functions on a locally compact Hausdorff space $X$. If $\mathfrak{C}$ has a multiplicative identity, $X$ is compact. So behind every abstract abelian $C^{*}$-algebra there is a classical phase space theory defined by this 'function representation' on the phase space $X$. Conversely, every classical phase space theory defines a $C^{*}$-algebra. For example, the observables of a classical system of $n$ particles—real-valued functions on the phase space $\mathbb{R}^{6 n}$-can be represented as the self-adjoint elements of the $C^{*}$-algebra $\mathfrak{B}\left(\mathbb{R}^{6 n}\right)$ of all continuous complex-valued functions $f$ on $\mathbb{R}^{6 n}$. The phase space $\mathbb{R}^{6 n}$ is locally compact and can be made compact by adding just one point 'at infinity,' or we can simply consider a closed and bounded (and thus compact) subset of $\mathbb{R}^{6 n}$. The statistical states of the system are given by probability measures $\mu$ on $\mathbb{R}^{6 n}$, and pure states, corresponding to maximally complete information about the particles, are given by the individual points of $\mathbb{R}^{6 n}$. The system's state $\rho$ in the $C^{*}$-algebraic sense is the expectation functional corresponding to $\mu$, defined by $\rho(f)=\int_{\mathbb{R}^{6 n}} f \mathrm{~d} \mu$.

So classical theories are characterized by commutative $C^{*}$-algebras. CBH identify quantum theories with a certain subclass of noncommutative $C^{*}$-algebras; specifically, theories where (i) the observables of the theory are represented by the self-adjoint operators in a noncommutative $C^{*}$-algebra (but the algebras of observables of distinct systems commute), (ii) the states of the theory are represented by $C^{*}$-algebraic states (positive normalized linear functionals on the $C^{*}$-algebra), and spacelike separated systems can be prepared in entangled states that allow what Schrödinger [31 p. 556] calls 'remote steering', and (iii) dynamical changes are represented by completely positive linear maps. For example, the standard quantum mechanics of a system with a finite number of degrees of freedom represented on a single Hilbert space with a unitary dynamics defined by a given Hamiltonian is a quantum theory, and theories with different Hamiltonians can be considered to be empirically inequivalent quantum theories. Quantum field theories for systems with an infinite number of degrees of freedom, where there are many unitarily inequivalent Hilbert space representations of the canonical commutation relations, are quantum theories. (For a detailed discussion and motivation for this identification, see [6, 77, 18, 19].)

What $\mathrm{CBH}$ showed was that one can derive the basic kinematic features of a quantumtheoretic description of physical systems in the above sense from three fundamental information-theoretic constraints: (i) the impossibility of superluminal information transfer between two physical systems by performing measurements on one of them, (ii) the impossibility of perfectly broadcasting the information contained in an unknown physical state (for pure states, this amounts to 'no cloning'), and (iii) the 
impossibility of communicating information so as to implement a certain primitive cryptographic protocol, called 'bit commitment,' with unconditional security. They also partly demonstrated the converse derivation, leaving open a question concerning nonlocality and bit commitment. This remaining issue has been resolved by Hans Halvorson [17], so we have a characterization theorem for quantum theory in terms of the three information-theoretic constraints.

To clarify the significance of the information-theoretic constraints, consider a composite quantum system $\mathrm{A}+\mathrm{B}$, consisting of two subsystems, A and B. For simplicity, assume the systems are identical, so their $C^{*}$-algebras $\mathfrak{A}$ and $\mathfrak{B}$ are isomorphic. The observables of the component systems $\mathrm{A}$ and $\mathrm{B}$ are represented by the self-adjoint elements of $\mathfrak{A}$ and $\mathfrak{B}$, respectively. Let $\mathfrak{A} \vee \mathfrak{B}$ denote the $C^{*}$-algebra generated by $\mathfrak{A}$ and $\mathfrak{B}$. The physical states of $\mathrm{A}, \mathrm{B}$, and $\mathrm{A}+\mathrm{B}$, are given by positive normalized linear functionals on their respective algebras that encode the expectation values of all observables (cf. standard quantum theory, where a state on $\mathfrak{B}(\mathcal{H})$ is defined by a density operator $D$ on $\mathcal{H}$ in terms of an expectation-valued functional $\rho(A)=\operatorname{Tr}(A D)$ for all observables represented by self-adjoint operators $A$ in $\mathfrak{B}(\mathcal{H})$.) To capture the idea that $\mathrm{A}$ and $\mathrm{B}$ are physically distinct systems, $\mathrm{CBH}$ make the assumption that any state of $\mathfrak{A}$ is compatible with any state of $\mathfrak{B}$, i.e., for any state $\rho_{A}$ of $\mathfrak{A}$ and $\rho_{B}$ of $\mathfrak{B}$, there is a state $\rho$ of $\mathfrak{A} \vee \mathfrak{B}$ such that $\left.\rho\right|_{\mathfrak{A}}=\rho_{A}$ and $\left.\rho\right|_{\mathfrak{B}}=\rho_{B}$.

The sense of the 'no superluminal information transfer via measurement' constraint is that when Alice and Bob, say, perform local measurements, Alice's measurements can have no influence on the statistics for the outcomes of Bob's measurements, and conversely. That is, merely performing a local measurement cannot, in and of itself, convey any information to a physically distinct system, so that everything 'looks the same' to that system after the measurement operation as before, in terms of the expectation values for the outcomes of measurements. $\mathrm{CBH}$ show that it follows from this constraint that A and B are kinematically independent systems if they are physically distinct in the above sense, i.e., every element of $\mathfrak{A}$ commutes pairwise with every element of $\mathfrak{B}$.

The 'no broadcasting' condition now ensures that the individual algebras $\mathfrak{A}$ and $\mathfrak{B}$ are noncommutative. Broadcasting is a process closely related to cloning. In fact, for pure states, broadcasting reduces to cloning. In cloning, a ready state $\sigma$ of a system $\mathrm{B}$ and the state to be cloned $\rho$ of system A are transformed into two copies of $\rho$. In broadcasting, a ready state $\sigma$ of B and the state to be broadcast $\rho$ of A are transformed to a new state $\omega$ of $A+B$, where the marginal states of $\omega$ with respect to both $A$ and $B$ are $\rho$. In elementary quantum mechanics, neither cloning nor broadcasting is possible in general. A pair of pure states can be cloned if and only if they are orthogonal and, more generally, a pair of mixed states can be broadcast if and only if they are represented by mutually commuting density operators. $\mathrm{CBH}$ show that broadcasting and cloning are always possible for classical systems, i.e., in the commutative case there is a universal broadcasting map that clones any pair of input pure states and broadcasts any pair of input mixed states. Conversely, they show that if any two states can be (perfectly) broadcast, then any two pure states can be cloned; and if two pure states of a $C^{*}$ algebra can be cloned, then they must be orthogonal. So, if any two states can be broadcast, then all pure states are orthogonal, from which it follows that the algebra is commutative.

The quantum mechanical phenomenon of interference is the physical manifestation of the noncommutativity of quantum observables or, equivalently, the superposition of quantum states. So the impossibility of perfectly broadcasting the information contained in an unknown physical state, or of cloning or copying the information in an unknown pure state, is the information-theoretic counterpart of interference.

Now, if $\mathfrak{A}$ and $\mathfrak{B}$ are noncommutative and mutually commuting algebras of observ- 
ables associated with distinct spatially separated systems, it can be shown that there are nonlocal entangled states on the $C^{*}$-algebra $\mathfrak{A} \vee \mathfrak{B}$ they generate (see [25, 34, 1], andmore relevantly here, in terms of a specification of the range of entangled states that can be guaranteed to exist-[17]). So it seems that entanglement-what Schrödinger [31. p. 555] called 'the characteristic trait of quantum mechanics, the one that enforces its entire departure from classical lines of thought' - follows automatically in any theory with a noncommutative algebra of observables. That is, it seems that once we assume 'no superluminal information transfer via measurement,' and 'no broadcasting,' the class of allowable physical theories is restricted to those theories in which physical systems manifest both interference and nonlocal entanglement. But this conclusion is surely too quick, since the derivation of entangled states depends on formal properties of the $C^{*}$-algebraic machinery. Moreover, we have no assurance that two systems in an entangled state will maintain their entanglement indefinitely as they separate in space, which is the case for quantum entanglement. But this is precisely what is required by the cheating strategy that thwarts secure bit commitment, since Alice will have to keep one system of such a pair and send the other system to Bob, whose degree of spatial separation from Alice is irrelevant, in principle, to the implementation of the protocol. In an information-theoretic characterization of quantum theory, the fact that entangled states of composite systems can be instantiated, and instantiated nonlocally so that the entanglement of composite systems is maintained as the subsystems separate in space, should be shown to follow from some information-theoretic principle. The role of the 'no bit commitment' constraint is to guarantee entanglement maintenance over distance, that is, the existence of a certain class of nonlocal entangled states-hence it gives us nonlocality, not merely 'holism.'

Bit commitment is a cryptographic protocol in which one party, Alice, supplies an encoded bit to a second party, Bob, as a warrant for her commitment to 0 or 1 . The information available in the encoding should be insufficient for Bob to ascertain the value of the bit at the initial commitment stage, but sufficient, together with further information supplied by Alice at a later stage when she is supposed to 'open' the commitment by revealing the value of the bit, for Bob to be convinced that the protocol does not allow Alice to cheat by encoding the bit in a way that leaves her free to reveal either 0 or 1 at will.

In 1984, Bennett and Brassard [3] proposed a quantum bit commitment protocol now referred to as BB84. The basic idea was to encode the 0 and 1 commitments as two quantum mechanical mixtures represented by the same density operator, $\omega$. As they showed, Alice can cheat by adopting an EPR attack or cheating strategy. Instead of following the protocol and sending a particular mixture to Bob she prepares pairs of particles A+B in the same entangled state $\rho$, where $\left.\rho\right|_{\mathfrak{B}}=\omega$. She keeps one of each pair (the ancilla A) and sends the second particle B to Bob, so that Bob's particles are in the mixed state $\omega$. In this way she can reveal either bit at will at the opening stage, by effectively steering Bob's particles into the desired mixture via appropriate measurements on her ancillas. Bob cannot detect this cheating strategy.

Mayers [28, 29], and Lo and Chau [26], showed that the insight of Bennett and Brassard can be extended to a proof that a generalized version of the EPR cheating strategy can always be applied, if the Hilbert space is enlarged in a suitable way by introducing additional ancilla particles. The proof of this 'no go' quantum bit commitment theorem exploits biorthogonal decomposition via a result by Hughston, Jozsa, and Wootters [21]. Informally, this says that for a quantum mechanical system consisting of two (separated) subsystems represented by the $C^{*}$-algebra $\mathfrak{B}\left(\mathcal{H}_{1}\right) \otimes \mathfrak{B}\left(\mathcal{H}_{2}\right)$, any mixture of states on $\mathfrak{B}\left(\mathcal{H}_{2}\right)$ can be generated from a distance by performing an appropriate POV-measurement on the system represented by $\mathfrak{B}\left(\mathcal{H}_{1}\right)$, for an appropriate entangled state of the composite system $\mathfrak{B}\left(\mathcal{H}_{1}\right) \otimes \mathfrak{B}\left(\mathcal{H}_{2}\right)$. Schrödinger [31, p. 556] 
called this 'remote steering' and found the possibility so physically counterintuitive that he speculated [32. p. 451] (wrongly, as it turned out) that experimental evidence would eventually show that this was simply an artifact of the theory, and that any entanglement between two systems would spontaneously (and instantaneously) decay as the systems separated in space. Remote steering is what makes it possible for Alice to cheat in her bit commitment protocol with Bob. It is easy enough to see this for the original BB84 protocol. Suprisingly, this is also the case for any conceivable quantum bit commitment protocol. (See Bub [5] for a discussion.)

Now, unconditionally secure bit commitment is also impossible for classical systems, in which the algebras of observables are commutative. ${ }^{1}$ But the insecurity of any bit commitment protocol in a noncommutative setting depends on considerations entirely different from those in a classical commutative setting. Classically, unconditionally secure bit commitment is impossible, essentially because Alice can send (encrypted) information to Bob that guarantees the truth of an exclusive classical disjunction (equivalent to her commitment to a 0 or a 1 ) only if the information is biased towards one of the alternative disjuncts (because a classical exclusive disjunction is true if and only if one of the disjuncts is true and the other false). No principle of classical mechanics precludes Bob from extracting this information. So the security of the protocol cannot be unconditional and can only depend on issues of computational complexity.

By contrast, the noncommutativity of quantum mechanics allows the possibility of different mixtures associated with the same density operator. If Alice sends Bob one of two mixtures associated with the same density operator to establish her commitment, then she is, in effect, sending Bob evidence for the truth of an exclusive disjunction that is not based on the selection of a particular disjunct (' 0 or 1 '). What thwarts the possibility of using the ambiguity of mixtures in this way to implement an unconditionally secure bit commitment protocol is the existence of nonlocal entangled states, and the maintenance of entanglement as entangled systems separate. This allows Alice to cheat by preparing a suitable entangled state instead of one of the mixtures, where the reduced density operator for Bob is the same as that of the mixture. Alice is then able to steer Bob's systems remotely into either of the two mixtures associated with the alternative commitments at will. (See Bub [6] for a further discussion.)

So what would allow unconditionally secure bit commitment in a noncommutative theory is the spontaneous decay of entangled states of composite systems as the component systems separate in space (specifically, the entangled state of the pair of systems prepared by Alice, one of which she sends to Bob). One can therefore take Schrödinger's remarks (with hingdsight) as relevant to the question of whether or not secure bit commitment is possible in our world. In effect, Schrödinger raised the possibility that we live in a quantum-like world in which secure bit commitment is possible! It follows that the impossibility of unconditionally secure bit commitment entails that, for any mixed state that Alice and Bob can prepare by following some (bit commit-

\footnotetext{
${ }^{1}$ Adrian Kent $[22$ has shown how to implement a secure classical bit commitment protocol by exploiting relativistic signalling constraints in a timed sequence of communications between verifiably separated sites for both Alice and Bob. In a bit commitment protocol, as usually construed, there is a time interval of arbitrary length, where no information is exchanged, between the end of the commitment stage of the protocol and the opening or unveiling stage, when Alice reveals the value of the bit. Kent's ingenious scheme effectively involves a third stage between the commitment state and the unveiling stage, in which information is exchanged between Bob's sites and Alice's sites at regular intervals until one of Alice's sites chooses to unveil the originally committed bit. At this moment of unveiling the protocol is not yet complete, because a further sequence of unveilings is required between Alice's sites and corresponding sites of Bob before Bob has all the information required to verify the commitment at a single site. If a bit commitment protocol is understood to require an arbitrary amount of 'free' time between the end of the commitment stage and the opening stage (in which no step is to be executed in the protocol), then unconditionally secure bit commitment is impossible for classical systems. (I am indebted to Dominic Mayers for clarifying this point.)
} 
ment) protocol, there is a corresponding entangled state that remains entangled as the component systems separate and pass between Alice and Bob.

To sum up: the content of the $\mathrm{CBH}$ theorem is that a quantum theory-a theory where (i) the observables of the theory are represented by the self-adjoint operators in a noncommutative $C^{*}$-algebra (but the algebras of observables of distinct systems commute), (ii) the states of the theory are represented by $C^{*}$-algebraic states (positive normalized linear functionals on the $C^{*}$-algebra), and spacelike separated systems can be prepared in entangled states that allow remote steering, and (iii) dynamical changes are represented by completely positive linear maps — can be characterized by the three information-theoretic 'no-go's': no superluminal communication of information via measurement, no (perfect) broadcasting, and no (unconditionally secure) bit commitment.

\section{Quantum Information}

The significance of the CBH theorem is that we can now see quantum mechanics as a principle theory, where the principles are information-theoretic constraints. A relativistic theory is a theory characterized by certain symmetry or invariance properties, defined in terms of a group of space-time transformations. Following Einstein's formulation of special relativity as a principle theory, we understand this invariance to be a consequence of the fact that we live in a world in which natural processes are subject to certain constraints. (Recall Einstein's characterization of the special principle of relativity as 'a restricting principle for natural laws, comparable to the restricting principle of the non-existence of the perpetuum mobile which underlies thermodynamics.') $\mathrm{CBH}$ treat a quantum theory as a theory in which the observables and states have a certain characteristic algebraic structure. So for $\mathrm{CBH}$, 'quantum' is a structural adjective applicable to theories, just as 'relativistic' is. Unlike relativity theory, quantum mechanics was born as a recipe or algorithm for caclulating the expectation values of observables measured by macroscopic measuring instruments. The interpretative problems arise because this Hilbert space theory has no phase space representation. Without Einstein's analysis, we could also see Minkowski space-time simply as an algorithm for relativistic kinematics and the Lorentz transformation, which is incompatible with the kinematics of Newtonian space-time. What Einstein's analysis provides is a rationale for taking the structure of space-time as Minkowskian: we see that this is required for the consistency of the two principles of special relativity. From this perspective, it is also clear that, insofar as a constructive theory like Lorentz's theory is constrained by the requirement to reproduce the empirical content of the principles of special relativity (which means that the ether as a rest frame for electromagnetic phenomena must, in principle, be undetectable), such a theory can have no excess empirical content over special relativity. Cushing [10 p. 193] quotes Maxwell as asking whether 'it is not more philosophical to admit the existence of a medium which we cannot at present perceive, than to assert that a body can act at a place where it is not.' Yes, but not if we also have to admit that, in principle, as a matter of physical law, if we live in a world in which events are constrained by the two relativistic principles, the medium must remain undetectable.

Consider again the transition:

Lorentz's constructive theory

$\longrightarrow$ special relativity as a principle theory (via Einstein's analysis)

$\longrightarrow$ Minkowski space-time

and the counterfactual history: 
Bohm's constructive theory

$\longrightarrow$ quantum mechanics as a principle theory (via $\mathrm{CBH}$ )

$\longrightarrow$ Hilbert space representation of states and observables

What the $\mathrm{CBH}$ analysis provides is a rationale for taking the structure of states and observables associated with quantum phenomena as a noncommutative $C^{*}$-algebra, represented on a Hilbert space with no phase space representation. From the $\mathrm{CBH}$ theorem, a theory satisfies the information-theoretic constraints if and only if it is empirically equivalent to a quantum theory (a theory where the observables, the states, and the dynamics are represented as outlined at the end of Section 3). So if the informationtheoretic constraints are satisfied, a constructive theory like Bohm's theory can have no excess empirical content over a quantum theory. Just as in the case of Lorentz's theory, Bohm's theory will have to posit contingent assumptions to hide the additional mechanical structures (the hidden variables will have to remain hidden), so that in principle, as a matter of physical law, there could not be any evidence favouring the theory over quantum theory.

Consider how this is achieved in Bohm's theory. The additional mechanical structures in Bohm's theory are the particle trajectories in configuration space, and the wave function as a guiding field. The dynamical evolution of a Bohmian particle is described by a deterministic equation of motion in configuration space that is guaranteed to produce the quantum statistics for all quantum measurements, if the initial distribution over particle positions (hidden variables) is the Born distribution. The Born distribution is treated as an equilibrium distribution, and non-equilibrium distributions can be shown to yield predictions that conflict with the information-theoretic constraints. Valentini [35] shows how non-equilibrium distributions can be associated with such phenomena as instantaneous signalling between spatially separated systems and the possibility of distinguishing nonorthogonal pure states (hence the possibility of cloning such states). Key distribution protocols whose security depends on 'no information gain without disturbance' and 'no cloning' would then be insecure against attacks based on exploiting such non-equilibrium distributions.

On Bohm's theory, the explanation for the fact that the information-theoretic constraints hold in our universe is that the universe has in fact reached the equilibrium state with respect to the distribution of hidden variables. But now it is clear that there can be no empirical evidence for the additional structural elements of Bohm's theory that would represent excess empirical content over a quantum theory, because such evidence is in principle unobtainable in the equilibrium state. If the information-theoretic constraints apply at the phenomenal level then, according to Bohm's theory, the universe must be in the equilibrium state, and in that case there can be no phenomena that are not part of the empirical content of a quantum theory (i.e., the statistics of quantum superpositions and entangled states). Since a similar analysis will apply to any 'no collapse' hidden variable theory-this, in effect, is what the 'no go' hidden variable theorems tell us: any such theory will have to incorporate the basic features of Bohm's theory - the additional non-quantum structural elements that these theories postulate cannot be doing any work in providing a physical explanation of quantum phenomena that is not already provided by an empirically equivalent quantum theory.

Of course, it could be the case that we are mistaken about the information-theoretic constraints, and that some day we will find experimental evidence that conflicts with the predictions of a quantum theory. The above claim about constructive theories like Bohm's theory is a conditional claim about what follows if the information-theoretic constraints do in fact hold in our world. To put the point differently: an acceptable mechanical theory of quantum phenomena that includes an account of our measuring instruments as well as the phenomena they reveal must violate at least one of the 
information-theoretic constraints.

What led to Lorentz's theory was a problem about the electromagnetic field, conceived as an aspect of the motion of a mechanical medium. The rejection of Lorentz's constructive theory in favour of Einstein's principle theory requires that we consider a field as a new physical primitive, not reducible to the motion of particles or a mechanical medium. What led to Bohm's theory was a problem about the difficulty of representing information from macroscopic classically described measuring instruments in a phase space theory that could account for the behaviour of the measuring instruments as well as the phenomena revealed by these instruments. If the $C^{*}$-algebra is commutative, there is a phase space representation of the theory-not necessarily the phase space of classical mechanics, but a theory in which the observables of the $C^{*}$-algebra are replaced by 'beables' (Bell's term, see [2]) or dynamical quantities, and the $C^{*}$ algebraic states are replaced by states representing complete catalogues of properties (idempotent quantities). In this case, it is possible to extend the theory to include the measuring instruments that are the source of the $C^{*}$-algebraic statistics, so that they are no longer 'black boxes' but constructed out of systems that are characterized by properties and states of the phase space theory. That is, the $C^{*}$-algebraic theory can be replaced by a 'detached observer' theory of the physical processes underlying the phenomena, to use Pauli's term [30], including the processes involved in the functioning of measuring instruments. Note that this depends on a representation theorem. In the noncommutative case, we are guaranteed only the existence of a Hilbert space representation of the $C^{*}$-algebra, and the possibility of a 'detached observer' description of the phenomena is a further question to be investigated.

In a review of Cushing's [10], di Salle [12] p. 755] quotes Pauli as remarking in his Theory of Relativity [30 p. vi] that the ether 'had to be given up, not only because it turned out to be unobservable, but because it became superfluous as an element of a mathematical formalism, the group-theoretical properties of which would only be disturbed by it.' Similarly, Pauli says, the concept of definite particle trajectories or space-time orbits had to be given up in quantum mechanics 'not only because the orbits are unobservable, but because they became superfluous and woud disturb the symmetry inherent in the general transformation group underlying the mathematical formalism of the theory.' DiSalle comments:

Evidently this is neither a simple empiricist rejection of the unobservable, nor an operationalist reduction of the meanings of theoretical terms to processes of measurement. Instead, it asserts that the purpose of any formalism in physics is to represent the known lawful behaviour of observable systems, and that distinctions or symmetries that don't belong to observable systems don't belong to their theoretical representation either. ... Thus it seems odd that Cushing should ask, 'What is it about the formalism of quantum mechanics that makes it so difficult to tell a story that we feel we understand about physical phenomena?' (p.341). The problem isn't with the formalism at all; the project of telling such a story is orthogonal to that of the formalism, which is to represent the structure of the physical world as it actually reveals itself to us. That structure may appear bizarre, but its bizarre aspects are necessarily incorporated into deterministic alternatives to quantum mechanics. Moreover, to regard the uncertainty relations as a kind of natural 'conspiracy' to hide the underlying determinism must have seemed, to Pauli et al., precisely as odd as accepting the Lorentz contraction instead of special relativity, and for precisely the same reason. So, instead of Cushing's question, one could ask, what is it about physical reality that makes it difficult to represent it by a 
deterministic theory? Why is it that any empirically adequate deterministic theory must be so constructed as to mimic an indeterministic theory in every conceivable empirical circumstance?

DiSalle's question is answered by the CBH characterization of quantum mechanics in terms of information-theoretic principles. The rejection of 'detached observer' hidden variable theories in favour of quantum mechanics requires that our measuring instruments ultimately remain black boxes at some level. That is, a quantum description will have to introduce a 'cut' between what we take to be the ultimate measuring instrument in a given measurement process and the quantum phenomenon revealed by the instrument, which means that the ultimate measuring instrument is treated simply as a probabilistic source of a range of labelled events or 'outcomes,' i.e., effectively as a source of information in Shannon's sense. But this amounts to treating quantum mechanics as a theory about the representation and manipulation of information constrained by the possibilities and impossibilities of information-transfer in our world (a fundamental change in the aim of physics), rather than a theory about the behavior of nonclassical waves and particles.

Something like this view seems to be implicit in Bohr's complementarity interpretation of quantum theory. For Bohr, quantum mechanics is complete and there is no measurement problem, but measuring instruments ultimately remain outside the quantum description: the placement of the 'cut' between system and measuring instrument is arbitrary, but the cut must be placed somewhere. Similarly, the argument here is that, if the information-theoretic constraints hold in our world, the measurement problem is a pseudo-problem, and the whole idea of an empirically equivalent 'interpretation' of quantum theory that 'solves the measurement problem' is to miss the point of the quantum revolution.

So a consequence of rejecting 'detached observer' hidden variable theories is that we recognize information as a new physical primitive, not reducible to the properties of particles or fields. An entangled state should be thought of as a new sort of nonclassical communication channel that we have discovered to exist in our universe, i.e., as a new sort of 'wire.' We can use these communication channels to do things that would be impossible otherwise, e.g., to teleport states, to compute in new ways, etc. Quantum theory is then about the properties of these communication channels, and about the representation and manipulation of states as sources of information in this physical sense.

The question: 'What is information in the physical sense (if it is not reducible to the properties of particles or fields)?' should be seen as like the question: 'What is a field in the physical sense (if it is not reducible to the motion of particles or a mechanical medium)?' The answer is something like this: Quantum mechanics represents the discovery that there are new sorts of information sources and communication channels in nature (represented by quantum states), and the theory is about the properties of these information sources and communication channels. You can, if you like, tell a mechanical story about quantum phenomena (via Bohm's theory, for example) but such a story, if constrained by the information-theoretic principles, can have no excess empirical content over quantum mechanics, and the additional non-quantum structural elements will be explanatorily superfluous. So the mechanical story for quantum phenomena is like an ether story for electromagnetic fields. Just as the ether story attempts to make sense of the behaviour of fields by proposing an ether that is a sort of sui generis mechanical system different from all other mechanical systems, so a Bohmian story attempts to make sense of quantum phenomena by introducing a field (the quantum potential or guiding field) that is a sort of sui generis field different from other 
physical fields. ${ }^{2}$

Cushing [10 p. 204] quotes Lorentz (from the conclusion of the 1916 edition of The Theory of Electrons) as complaining that 'Einstein simply postulates what we have deduced.'

I cannot speak here of the many highly interesting applications which Einstein has made of this principle [of relativity]. His results concerning electromagnetic and optical phenomena ... agree in the main with those which we have obtained in the preceding pages, the chief difference being that Einstein simply postulates what we have deduced, with some difficulty and not altogether satisfactorily, from the fundamental equations of the electromagnetic field. By doing so, he may certainly take credit for making us see in the negative result of experiments like those of Michelson, Rayleigh and Brace, not a fortuitous compensation of opposing effects, but the manifestation of a general and fundamental principle.

Yet, I think, something may also be claimed in favour of the form in which I have presented the theory. I cannot but regard the aether, which can be the seat of an electromagnetic field with its energy and its vibrations, as endowed with a certain degree of substantiality, however different it may be from all ordinary matter. In this line of thought, it seems natural not to assume at starting that it can never make any difference whether a body moves through the aether or not, and to measure distances and lengths of time by means of rods and clocks having a fixed position relative to the aether.

Similarly, one might complain that CBH simply postulate what is ultimately explained by a Bohmian (or other 'no collapse') theory. Just as the rejection of Lorentz's complaint involves taking the field as a new physical primitive (tantamount to 'no ether,' which follows once we accept the principles of special relativity as basic to an explanatory account of electromagnetic phenomena), so the rejection of the analogous complaint in the quantum case involves taking information as a new physical primitive (tantamount to 'measuring instruments are ultimately to be treated as black boxes,' which follows-via the 'no go' theorems-once we accept the three information-theoretic constraints as basic to an explanatory account of quantum phenomena).

To conclude, it might be worthwhile clarifying what is not being argued here. Firstly, the CBH theorem should not be understood as providing a 'constructive' explanation for the quantum formalism, along the lines suggested by Chris Fuchs [15] (or the axiomatization proposed by Lucien Hardy [20], or by quantum logicians), but rather as a 'principled' reconstruction of the theory within a suitably general mathematical framework. Secondly, the claim that quantum mechanics is about quantum information - that quantum mechanics is a principle theory of information (in the sense in which Einstein regarded special relativity as a principle theory) — and that this physical notion of information is not reducible to the properties of particles or fields, is not to be construed as the claim that quantum mechanics is about observers and their epistemological concerns, nor that we have derived 'it from bit' in Wheeler's sense [36] of a 'participatory universe,' nor that the basic stuff of the world is informational in an intentional sense. (Recall Shannon's remark [33 p.31] that 'the semantic aspects of communication are irrelevant to the engineering problem'-it is the 'engineering' sense of information that is relevant to the $\mathrm{CBH}$ theorem.) Rather, the claim is that the

\footnotetext{
${ }^{2}$ In fact, in [4] section 3.2] Bohm and Hiley suggest that the guiding field should be understood as a sort of informational field.
} 
lesson of modern physics is that a principle theory is the best one can hope to achieve as an explanatory account of quantum phenomena.

\section{Acknowledgements}

The ideas in this paper originated during a research leave supported by a University of Maryland Sabbatical Leave Fellowship and a General Research Board Fellowship in 2001-2002.

\section{References}

[1] G. Bacciagaluppi, 'Separation theorems and Bell inequalities in algebraic quantum mechanics,' in P. Busch, P. Lahti, and P. Mittelstaedt (eds.), Symposium on the Foundations of Modern Physics 1993: Quantum Measurement, irreversibility and the Physics of Information (Singapore: World Scientific, 1994), (pp. 29-37).

[2] J. S. Bell, 'Beables for quantum field theory,' in J.S. Bell, Speakable and Unspeakable in Quantum Mechanics, pp. 173-180 (Cambridge: Cambridge University Press: 1987).

[3] C.H. Bennett and G. Brassard, 'Quantum cryptography: public key distribution and coin tossing,' in Proceedings of IEEE International Conference on Computers, Systems, and Signal Processing, pp. 175-179 (New York: IEEE, 1984).

[4] D. Bohm and B.J. Hiley, B., The Undivided Universe: An Ontological Interpretation of Quantum Theory (London: Routledge, 1993).

[5] J. Bub, 'The bit commitment theorem,' Foundations of Physics 31, 735-756 (2001).

[6] J. Bub, 'Why the Quantum?' Studies in the History and Philosophy of Modern Physics 35, 241-266 (2004).

[7] R. Clifton, J. Bub, and H. Halvorson, 'Characterizing quantum theory in terms of information-theoretic constraints,' Foundations of Physics 33, 1561-1591 (2003).

[8] A. Connes, Noncommutative Geometry (Academic Press, San Diego, 1994).

[9] James T. Cushing, Quantum Mechanics: Historical Contingency and the Copenhen Hegemony (Chicago: University of Chicago Press, 1994).

[10] James T. Cushing, Philosophical Concepts in Physics (Cambridge: Cambridge University Press, 1998).

[11] James T. Cushing, Arthur Fine, and Sheldon Goldstein (eds.), Bohmian Mechanics and Quantum Theory: An Appriaisal (Dordrecht: Kluwer, 1996).

[12] R. DiSalle, Review of James T. Cushing: Philosophical Concepts in Physics: The Historical Relation between Philosophy and Scientific Theories, British Journal for Philosophy of Science 50, 747-759 (1999).

[13] A. Einstein, "What is the theory of relativity." First published in The Times, London, November 28, 1919, p. 13. Also in A. Einstein, Ideas and Opinions (New York: Bonanza Books, 1954), pp. 227-232. 
[14] A. Einstein, 'Autobiographical notes,' in Albert Einstein: Philosopher-Scientist, P.A. Schilpp, ed. (La Salle, IL: Open Court, 1949).

[15] C.A. Fuchs, 'Quantum mechanics as quantum information (and only a little more),' in A. Khrenikov (ed.) Quantum Theory: Reconstruction of Foundations (Växjo: Växjo University Press, 2002).

[16] S. Goldstein, 'Bohmian mechanics,' in E.N. Zalta (ed.), Stanford Encyclopedia of Philosophy. http://plato.stanford.edu/entries/qm-Bohm

[17] H. Halvorson, 'Generalization of the Hughston-Jozsa-Wootters theorem to hyperfinite von Neumann algebras,' quant-ph/031001.

[18] H. Halvorson, 'A note on information-theoretic characterizations of physical theories,' quant-ph/0310101

[19] H. Halvorson and J. Bub, 'Can quantum cryptography imply quantum mechanics? Reply to Smolin,' quant-ph/0311065

[20] L. Hardy, 'Quantum theory from five reasonable axioms,' quant-ph/0101012 and 'Why quantum theory,' in J. Butterfield and T. Placek (eds.), Proceedings of the NATO Advanced Research Workshop on Modality, Probability, and Bell's Theorem (Amsterdam: IOS Press, 2002).

[21] L.P. Hughston, R. Jozsa, and W.K. Wootters, 'A complete classification of quantum ensembles having a given density matrix,' Physics Letters A 183, 14-18 (1993).

[22] A. Kent, 'Unconditionally secure bit commitment,' Physical Review Letters 83, 1447-1450 (1999).

[23] M.J. Klein, 'Thermodynamics in Einstein's thought,' Science 157, 509-516 (1967).

[24] N. Landsman, Mathematical Topics Between Classical and Quantum Mechanics (New York: Springer, 1998)

[25] L.J. Landau, 'On the violation of Bell's inequality in quantum theory,' Physics Letters A 120, 54-56 (1987).

[26] H.-K. Lo and H.F. Chau, 'Is quantumbBit commitment really possible?', Physical Review Letters 78, 3410-3413 (1997).

[27] H.A. Lorentz, The Theory of Electrons (Columbia University Press, New York, 1909).

[28] D. Mayers, 'Unconditionally secure quantum bit commitment is impossible,' in Proceedings of the Fourth Workshop on Physics and Computation (New England Complex System Institute, Boston, 1996), pp. 224-228.

[29] D. Mayers, 'Unconditionally secure quantum bit commitment is impossible,' Physical Review Letters 78, 3414-3417 (1997).

[30] W. Pauli, letter to M. Born dated March 30, 1954, in M. Born (ed.), The BornEinstein Letters, p. 218 (London: Walker and Co: 1954).

[31] E. Schrödinger, 'Discussion of probability relations between separated systems,' Proceedings of the Cambridge Philosophical Society 31, 555-563 (1935). 
[32] E. Schrödinger, 'Probability relations between separated systems,' Proceedings of the Cambridge Philosophical Society 32, 446-452 (1936).

[33] C.E. Shannon, 'The mathematical theory of communication,' Bell System Technical Journal 27, 379-423, 623-656 (1948); reprinted in C.E. Shannon and W. Weaver, The Mathematial Theory of Communication (University of Illinois Press, Urbana, 1963), pp. 30-125.

[34] S. Summers, 'On the independence of local algebras in quantum field theory,' Reviews in Mathematical Physics, 2, 201-247 (1990).

[35] A. Valentini, 'Subquantum information and computation,' quant-ph/0203049.

[36] J.A. Wheeler, 'Information, physics, quantum: the search for links,' in W.H. Zurek (ed.), Complexity, Entropy, and the Physics of Information (Redwood City, CA: Addison-Wesley, 1990), pp. 3-28. 